\title{
EARLIEST SYMPTOMS OF PRESENTATION IN COVID-19 PATIENTS IN TERTIARY CARE HOSPITAL
}

\author{
Maqbool Raza, Muhammad Ali Raza*, De Emmal Asjad Cheema**, Maham Asjad Cheema*, Atif Rafique, Sarim Waleed Khan*** \\ Combined Military Hospital Multan/National University of Medical Sciences (NUMS) Pakistan, *Islamabad Medical and Dental College, Islamabad Pakistan, \\ **Pakistan Medical Commission, Islamabad Pakistan, ${ }^{* * *}$ HBS Medical and Dental College, Islamabad Pakistan
}

\section{ABSTRACT}

Objective: To determine the frequency of earliest symptoms of COVID-19 infection among patients with confirmed SARSCOVID-19 infection.

Study Design: Cross-sectional analytical study.

Place and Duration of Study: Combined Military Hospital Multan, from Jun to Dec 2021.

Methodology: Data from 299 patients admitted in tertiary care settings was collected on a questionnaire. Patients regardless of gender and age who had confirmed COVID-19 infection through Real Time Polymerase Chain Reaction (RT-PCR) were included in the study. A nonprobability consecutive sampling technique was used to select samples. Data was entered and analyzed through SPSS version 22. Frequencies and percentages of various presenting symptoms were calculated. Sample size calculated at $95 \%$ level of confidence, $1 \%$ required precision, and $27 \%$ anticipated population proportion were 299 . The overall difference in frequencies of symptoms in various groups was compared by using chi-square test. $p$-value $<0.05$ was taken as significant.

Results: A total of 299 participants were included in this analysis. The median age for participants (interquartile range [IQR]) was 46 (36-54) years. Among 299 adults the reported symptoms were cough 238 (79.6\%), fever 176 (58.7\%) and, dyspnea $113(37.8 \%)$. Only $78(26.1 \%)$ of participants with confirmed infection reported having all three symptoms of cough, fever, and dyspnea. Other reported symptoms in patients were diarrhea 54 (18.1\%), fatigue $128(42.8 \%)$, myalgia $113(37.8 \%)$, and anosmia 98 (32.8\%). There was no significant difference in the frequency of symptoms across both genders.

Conclusion: The most frequent symptoms of COVID-19 are cough, fever, and dyspnea.

Keywords: COVID-19, Cough, Fever, Gender.

How to Cite This Article: Raza M, Raza MA, Cheema DEA, Cheema MA, Rafique A, Khan SW. Earliest Symptoms of Presentation in COVID-19 Patients in Tertiary Care Hospital. Pak Armed Forces Med J 2021; 71(Suppl-3): S500-503. D Doi: https://doi.org/10.51253/pafmj.v1i1.7941

This is an Open Access article distributed under the terms of the Creative Commons Attribution License (https://creativecommons.org/licenses/by-nc/4.0/), which permits unrestricted use, distribution, and reproduction in any medium, provided the original work is properly cited.

\section{INTRODUCTION}

COVID-19 is a respiratory disease that is caused by the SARS COV-2 infection. The first case of Coronavirus disease 2019 (COVID-19) was reported in December 2019 in Wuhan, China. ${ }^{1}$ COVID-19 was declared a worldwide pandemic in March 2020 by The World Health Organization (WHO). ${ }^{2}$ Most commonly affected system by COVID-19 is the pulmonary system but it may affect both the central and peripheral nervous systems and gastro-Intestinal System.3.4 Mao et al, reported that $33 \%$ of patients with COVID-19 present neurologic symptoms and these were more common in patients with severe infection. ${ }^{4}$

The majority of patients with COVID-19 have mild to moderate respiratory disease while some people may experience severe disease, for example, COVID19 pneumonia. Accurate diagnosis of COVID-19 requires laboratory facility and Polymerase chain reaction (PCR) of nose and throat swabs, or imaging tests like CT scans. However, the first and most cost-effective

Correspondence: Dr Maqbool Raza, Assistant Professor of ENT, Classified ENT Specialist, Combined Military Hospital Multan Pakistan way to diagnose the infection is by identifying early symptoms and signs from clinical assessment. If the diagnosis is accurate through identifying the early symptoms and signs, the requirement for time-consuming, specialist diagnostic tests would be diminished. 5-7 Manifestations experienced by the patients suffering from mild COVID-19 may include dry cough, sore throat, high temperature, loose motion, headache, muscular or joint pain, fatigue, and loss of smell and taste. Manifestations of COVID-19 pneumonia may include shortness of breath, loss of appetite, confusion, pain or pressure in the chest, and high temperature (over $\left.38^{\circ} \mathrm{C}\right) .8,9$

Management of patients with COVID-19 depends on their symptoms and signs, patients with mild disease may be sent home for isolation and people with severe illness may receive further tests or be hospitalized. Accurate diagnosis is necessary so that it can be ensured that patients should receive the correct treatment promptly, and unnecessary tests, treatment and isolation of unaffected individuals be avoided. Accurate diagnosis is not only important for saving 
time and resources but also limiting the spread of disease. ${ }^{10}$ Pakistan is already suffering from the double burden of communicable and non-communicable diseases. As the COVID-19 pandemic unfolds, it is important to keep this in consideration as these highly prevalent endemic diseases may make people of the country more susceptible to severe COVID-19 infection. Information about SARS-CoV-2 infection, early symptoms, its transmission, the pathogenesis of the disease, modalities of diagnosis should be easily available and widespread. Considering the high risk of infection and the large number of individuals who may be carrying the virus in Pakistan it is of utmost impottance to recognize the early symptoms of COVID-19 so that isolation of these individuals before confirmatory diagnostic tests should limit the further spread of disease. This study was planned to determine the frequency of the earliest symptoms of COVID-19 in a tertiary care hospital.

\section{METHODOLOGY}

This cross-sectional analytical study was conducted in Combined Military Hospital Multan from June to December 2020 after taking the ethics approval from the Institutional ethical review committee. Sample size calculated at $95 \%$ level of confidence, $1 \%$ required precision, and $27 \%$ anticipated population proportion (Fever) were 299. Patients regardless of gender and age who had confirmed COVID-19 infection through RTPCR were included in the study. Patients who were suffering from severe disease (Oxygen-dependent) and on ventilator support were excluded. A nonprobability consecutive sampling technique was used to select the study subjects. Information was collected on a questionnaire containing the earliest symptoms of COVID19. The questionnaire comprising of 2 parts. The first part of the questionnaire contains general information regarding their demographics and the second part consist of survey questions assessing the early symptoms of the infection. Data was entered and analyzed through SPSS version 22. Mean and standard deviation was calculated for quantitative variable in the study like age. Frequencies and percentages of various presenting symptoms were calculated. Effect modifiers were controlled through stratification during data analysis. The overall difference in frequencies of symptoms in various groups was compared by using chi-square test. $p$ value $<0.05$ was taken as significant.

\section{RESULTS}

A total of 299 participants were included in this analysis. All participants with confirmed infection in this analysis had at least one symptom at the time of screening. The median age for the sample population (interquartile range [IQR]) was 46 (36-54) years. Among 299 adults with confirmed SARS-CoV-2 infection, $238(79.6 \%)$ reported cough, $176(58.7 \%)$ reported fever and $113(37.8 \%)$ reported dyspnea, irrespective of the time from symptom development. Only 78 (26.1\%) of participants with confirmed infection reported having all three symptoms of cough, fever, and dyspnea, while $151(50.5 \%)$ of participants had both fever and cough.

Other reported symptoms in patients with confirmed SARS-CoV-2 infection were diarrhea 54 (18.1\%), fatigue $128(42.8 \%)$, myalgia $113(37.8 \%)$, and anosmia 98 (32.8\%). To further explore the question of symptoms in SARS-CoV-2 infection, we examined reported rates of symptoms in participants with confirmed infection according to their gender. There was no significant difference in the frequency of symptoms across both genders.

Table-I: Gender distribution of the patients with confirmed SARS-CoV-2 infection

\begin{tabular}{l|c|c}
\hline Gender & Frequency & Percentage \\
\hline Male & 208 & $69.6 \%$ \\
\hline Female & 223 & $30.4 \%$ \\
\hline Total & 299 & $100 \%$ \\
\hline $\begin{array}{l}\text { Table-II: Earliest symptoms reported } \\
\text { confirmed SARS-CoV-2 infection }\end{array}$ \\
\hline Symptoms & Frequency & Percentage \\
\hline Cough & 238 & $79.6 \%$ \\
\hline Fever & 176 & $58.7 \%$ \\
\hline Dyspnea & 113 & $37.8 \%$ \\
\hline Cough, Fever \& Dyspnea & 78 & $26.1 \%$ \\
\hline Fever \& Cough & 151 & $50.5 \%$ \\
\hline Diarrhea & 54 & $18.1 \%$ \\
\hline Fatigue & 128 & $42.8 \%$ \\
\hline Myalgia & 113 & $37.8 \%$ \\
\hline
\end{tabular}

Table-III: Gender distribution and earliest symptoms reported by patients with confirmed SARS-CoV-2 infection.

\begin{tabular}{l|c|c}
\hline \multirow{2}{*}{ Symptoms } & \multicolumn{2}{|c}{ Gender } \\
\cline { 2 - 3 } & Male & Female \\
\hline Cough & $158(76 \%)$ & $65(65.6 \%)$ \\
\hline Fever & $75(36.1 \%)$ & $33(36.3 \%)$ \\
\hline Dyspnea & $68(33 \%)$ & $29(31.9 \%)$ \\
\hline Cough, Fever \& Dyspnea & $56(26.9 \%)$ & $25(27.5 \%)$ \\
\hline Fever \& Cough & $102(49 \%)$ & $46(50.5 \%)$ \\
\hline Diarrhea & $39(18.7 \%)$ & $16(17.65)$ \\
\hline Fatigue & $85(40.9 \%)$ & $40(44 \%)$ \\
\hline Myalgia & $71(38 \%)$ & $32(35.2 \%)$ \\
\hline
\end{tabular}

\section{DISCUSSION}

In the absence of widespread testing, symptomatic monitoring efforts may allow for understanding the epidemiological situation of the spread of coronavirus 
disease 2019 (COVID-19) in Pakistan. We obtained data from patients with confirmed SARS-CoV-19 infection admitted to the hospital to determine the frequency of COVID-19 related early symptoms. We analyzed data earliest COVID-19 related symptoms. Our study findings revealed that cough, fever, and dyspnea are the most frequent symptoms of COVID-19 which is consistent with findings of an early study in which cough, fever, and dyspnea have been described as the three most common symptoms of SARS-CoV-2 infection and these symptoms have also been promoted in guidelines as potential screening symptoms when deciding to test or not test a patient.11,12,21 The findings of the study showed that about $70 \%$ of participants with confirmed SARS-CoV-19 infection were male and there was no significant difference in earliest symptoms in both genders which is in line with the findings of Sharma et al in which the majority of the patients with COVID-19 were males and females seems to be protected against the infection. ${ }^{13}$

This generation belongs to a technology-oriented era in which social media, and smartphones play important role in creating awareness among the masses, on the contrary, they cannot be easily reached through traditional media like radio, newspaper, and television. It is the need of the hour to take COVID-19 seriously and share trustworthy. The use of social media may play a vital role in creating awareness regarding the prevention of SARS-CoV-19 infection and notifying the early symptoms of infection so that timely testing for confirmation and isolation of the patients may limit the further spread in the community. 14-16

The government of Pakistan is trying to spread awareness about the symptoms of disease and prevention strategies especially focusing on staying at home and maintaining social distance. Health authorities need to pay attention to educating the people for identification of early symptoms of COVID-19 and seriousness of the disease so that the false information among the public that the threat of coronavirus has been exaggerated is minimized. Government must encourage Television hosts to invite health care specialists in their programs and can run media campaigns in which correct information about symptoms is given to the public so that unnecessary burden on already an overburdened system can be reduced. ${ }^{17-19}$

The strength of the present study is that it tried to identify the earliest symptoms of COVID-19 in our local population. This is the basic information that must be known by the public. This information can then be used to begin early treatment in the population more sustainable to developing life-threatening symptoms and curbing the spread of infection. The study conducted helps bring to light the symptomatic differences based on the gender of the patient who is COVID-19 positive. This information opens opportunities for numerous researches in the future.

\section{ACKNOWLEDGEMENT}

The completion of this research would not have been possible without the participation and assistance of so many people whose names may not all be enumerated. Their contributions are sincerely appreciated and gratefully acknowledged. However, the group would like to express their deep appreciation and indebtedness particularly to the following. The ENT Department CMH Multan for their assistance during the research with patient records, examination and data collection.

The flu screening desk for their cooperation and for providing with data regarding patient symptoms. The Pathology department CMH Multan for providing prompt COVID PCR results so as to accommodate us with a good pace for our research.

\section{LIMITATION TO STUDY}

The study also has many limitations. The first is the participants were hospitalized in a tertiary care setting with a limited sample size that may lead to selection bias i.e., does not include data on people who do not have access to teaching hospitals. Therefore, it is difficult to verify the selection bias because it is not possible to evaluate the utilization rate of users as well as the demographics of patients not admitted to tertiary care hospitals. Secondly due to the limited sample size generalizability of the data is compromised.

\section{CONCLUSION}

The most frequently reported symptoms in patients with confirmed SARS-CoV-19 infection through PCR test are cough, fever, and dyspnea.

\section{Conflict of Interest: None.}

\section{Author's Contribution}

MR: Conceptualization, write-up, data collection, statistical analysis, proof reading, MAR: Data collection, data entry and analysis, DEAC: Literature search, write up, MAC: Data collection, data entry and analysis, AR: Literature search, proof reading, SWK: Data collection.

\section{REFERENCES}

1. Team E. The epidemiological characteristics of an outbreak of 2019 novel coronavirus diseases (COVID-19)-China, 2020. China CDC weekly 2020; 2(8): 113-115.

2. Velavan TP, Meyer CG. The COVID-19 epidemic. Tropical Med Inter Health 2020; 25(3): 278-282.

3. Wang D, Hu B, Hu C, Zhu F, Liu X, Zhang J, et al. Clinical characteristics of 138 hospitalized patients with 2019 novel coronavirus-infected pneumonia in Wuhan, China. J Am Med Assoc 2020; 323(11): 1061-1069. 


\section{Earliest Symptoms of COVID-19 Infection}

4. Mao L, Jin H, Wang M, Hu Y, Chen S, He Q, et al. Neurologic manifestations of hospitalized patients with coronavirus disease 2019 in Wuhan, China. JAMA Neurol 2020; 77(6): 683-690.

5. Elibol E. Otolaryngological symptoms in COVID-19. Europ Arch Oto-Rhino-Laryngol 2021; 278(4): 1233-1236.

6. Zhou Z, Zhao N, Shu Y, Han S, Chen B, Shu X. Effect of gastrointestinal symptoms in patients with COVID-19. Gastroenterol 2020; 158(8): 2294.

7. Sudre CH, Murray B, Varsavsky T, Graham MS, Penfold RS, Bowyer RC, et al. Attributes and predictors of long COVID. Nature Med 2021; 27(4): 626-631.

8. Çalıca Utku A, Budak G, Karabay O, Güçlü E, Okan HD, Vatan A. Main symptoms in patients presenting in the COVID19 period. Scottish Med J 2020; 65(4): 127-132.

9. Li J, Chen Z, Nie Y, Ma Y. Identification of symptoms prognostic of COVID-19 severity: multivariate data analysis of a case series in Henan Province. J Med Inter Res 2020; 22(6): e19636.

10. Han C, Duan C, Zhang S, Spiegel B, Shi H, Wang W, et al. Digestive symptoms in COVID-19 patients with mild disease severity: clinical presentation, stool viral RNA testing, and outcomes. Am J Gastroenterol 2020; 2020; 115(6): 916-923.

11. Mori M, Ikeda N, Taketomi A, Asahi Y, Takesue Y, Orimo T, et al. COVID-19: clinical issues from the Japan Surgical Society. Surg 2020; 1(1): 1-5.

12. Zachariah P, Johnson CL, Halabi KC. Epidemiology, clinical features, and disease severity in patients with coronavirus disease 2019 (COVID-19) in a child-ren's hospital in New York City, New York. JAMA Pediat 2020; 174(10): e202430.
13. Sharma G, Volgman AS, Michos ED. Sex differences in mortality from COVID-19 pandemic: are men vulnerable and women protected?. Case Reports 2020; 2(9): 1407-1410.

14. Williams KC, Page RA. Marketing to the generations. J Behav Stud Busin 2011; 3(1): 37-53.

15. Goel A, Gupta L. Social media in the times of COVID-19. J Clin Rheumatol 2020; 3(3): E175-194.

16. Bao H, Cao B, Xiong Y, Tang W. Digital media's role in the COVID-19 pandemic. JMIR Health Health 2020; 8(9): e20156.

17. Malik S. Knowledge of COVID-19 symptoms and prevention among Pakistani adults: a cross-sectional descriptive study. [Internet] Available from: file:///C:/Users/pafmj.amc/Downloads/Knowledge \%20of \%20COVID-19\%20Symptoms $\% 20$ and $\%$ 20Prevention\% 20among\%20Pakistani\%20Adults.pdf

18. Manigandan S, Wu MT, Ponnusamy VK, Raghavendra VB, Pugazhendhi A, Brindhadevi K. A systematic review on recent trends in transmission, diagnosis, prevention and imaging features of COVID-19. Pro Bioch 2020; 98(2): 233-240.

19. Kaleemi R, Hilal K, Arshad A, Martins RS, Nankani A, Tu H, et al. The association of chest radiographic findings and severity scoring with clinical outcomes in patients with COVID-19 presenting to the emergency department of a tertiary care hospital in Pakistan. PLoS One 2021; 16(1): e0244886.

20. Khan M, Khan H, Khan S, Nawaz M. Epidemiological and clinical characteristics of coronavirus disease (COVID-19) cases at a screening clinic during the early outbreak period: a single-centre study. J Med Microbiol 2020; 69(8): 1114-1118. 